favorable conditions a wave-length of $0.8 \mathrm{~mm}$. has been observed. The known electric wave spectrum has thus been extended toward shorter waves by an interval of from two to three octaves.

\title{
THE OPTICAL CONSTANTS OF CERTAIN LIQUIDS FOR SHORT ELECTRIC WAVES.
}

\author{
By J. D. Tear.
}

[ABSTRACT.]

In crossing the hitherto unexplored region of the spectrum extending from the extreme infra-red to the shortest electric waves, the reflection and absorption coefficients and the indices of refraction of a number of liquids undergo unusual changes. A notable example is water which for $\lambda 0.3 \mathrm{~mm}$. has an index near that for the visible region of the spectrum, but for $\lambda 30 \mathrm{~mm}$. has a refractive index of 8.5 , which is very nearly that for an infinite wave-length, i.e., the square root of the dielectric constant. Experiments, described in the preceding abstract, ${ }^{1}$ are now in progress which, it is hoped, will ultimately make a more complete exploration of this region possible. With the present equipment and methods the optical constants of a number of liquids, i.e., water, glycerin, methyl and ethyl alcohol for the wave-lengths 4, 8, I I, I8, and $27 \mathrm{~mm}$., have been determined.

The refractive indices have been computed by the CauchyQuincke formula from measured values of the coefficients of reflection and absorption. A second approximate, though direct determination of the refractive index, has been obtained by measuring the combined reflection from both surfaces of a thin liquid layer floated on mercury. As the thickness of the layer is varied, interference effects cause the reflected energy to pass through a series of maxima and minima which give a direct measurement of the wave-length in the liquid which divided into the wavelength in air gives the refractive index. The results obtained by these two methods are in good agreement.

The Thermal Stresses in Solid and in Hollow Circular Cylinders Concentrically Heated. Charles H. Lees. (Proc. Royal Soc., A 712 ).- " The large differences of temperature which exist between the inside and outside of the heating or firing tunnels used in pottery and

\footnotetext{
${ }^{1}$ E. F. Nichols and J. D. Tear, "Short Elecrtic Waves."
} 